\title{
V2X Communications for Platooning: Sensor Inaccuracy Aspects
}

\author{
Michał Sybis, Paweł Sroka, and Paweł Kryszkiewicz \\ Faculty of Electronics and Telecommunications, Poznan University of Technology, Poznan, Poland
}

https://doi.org/10.26636/jtit.2020.142820

\begin{abstract}
Platooning is a future approach to autonomous driving in which vehicle-to-vehicle and vehicle-to-infrastructure communications play an important role. Tests performed in the past showed that a significant reduction in fuel consumption is possible when cars are traveling in a dense platoon formation. To increase the level of their awareness of the surrounding objects and to maintain a very short distance to the preceding vehicle, highly reliable on-board sensors are required. This paper discusses the impact of sensor inaccuracy on the performance and behavior of and autonomous vehicle platoon that makes use of wireless communications supported by context information from various databases and maps.
\end{abstract}

Keywords-context information database, sensor accuracy, V2X and $5 G$.

\section{V2X Communications for Autonomous Driving}

The fifth generation $(5 \mathrm{G})$ wireless system is planned to provide, in the future, ultra-reliable low-latency communications (URLLC) that are necessary to enable various safetyrelated or life-saving services [1]. One of the numerous ideas behind URLLC, is to use it for the provision of highly reliable services, such as vehicle-to-vehicle (V2V) communications, that may be relied upon in the management of autonomous and driverless platoons formed by vehicles on high-speed roads. Autonomous driving is perceived to be one of the enablers for a coordinated movement of a group driverless vehicles, led by a platoon leader (platooning). In such a scenario, a group of vehicles exchange various control messages, enabling their autonomous movement at short inter-vehicle distances, without any human supervision. Increased interest in platooning is sparked primarily by the expected revenues, e.g. fuel savings of 7 to $15 \%$ for a platoon comprising multiple trucks [2]. Fuel savings result also in a substantial reduction of $\mathrm{CO}_{2}$ emissions according to the findings of the Energy project by ITS [3], if truck platooning was used in approximately $40 \%$ of all freight traffic operations, a $2.1 \%$ reduction in $\mathrm{CO}_{2}$ emissions along highways could be achieved with the gap be- tween trucks of $10 \mathrm{~m}$, and the reduction would equal $4.8 \%$ if the gap could be decreased even further to $4 \mathrm{~m}$.

Autonomous driving, aiming to improve road safety, may be achieved only with the support of both onboard sensors and inter-vehicular communications. However, to ensure that the automated controller responds faster than a human driver, extreme requirements concerned with reliability of wireless communications, as well as with the quality of on-board sensors used need to be met. For vehicle-toeverything (V2X) communications, the exchange of information between the platoon members and between the platoon and other devices may rely on short-range wireless communication protocols, such as dedicated short-range communications (DSRC), cooperative intelligent transport system (C-ITS) or cellular-V2X (CV2X). On the other hand, onboard sensors are necessary to provide information related to specific mobility parameters, facilitating the operation of autonomous vehicles. The accuracy of sensors used in platooning may cause various levels of deviations that strictly depend on the class of the physical devices installed. In this paper we consider a scenario that is depicted in Fig. 1, where the road train is driving, autonomously, on a high-speed road, in various environments. Stability and reliability are the most important aspects affecting intraplatoon communications. Onboard sensors also need to provide stable results that enable real-time and reliable

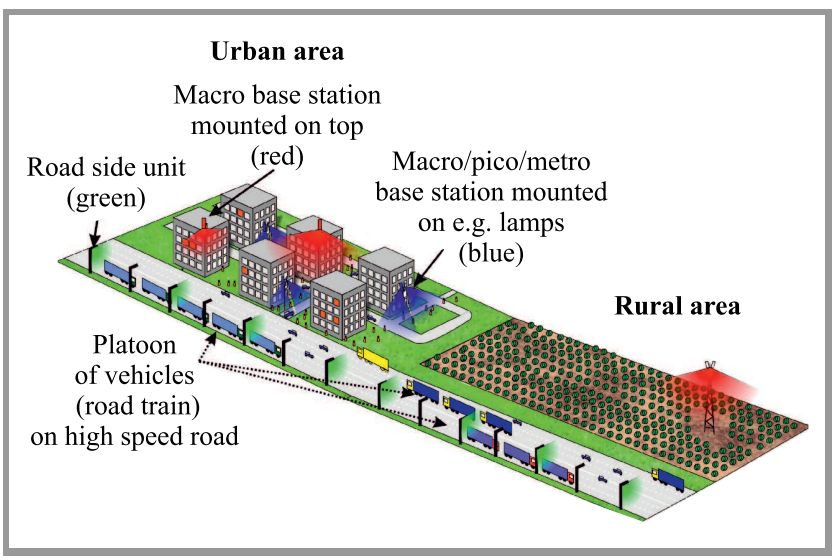

Fig. 1. Vehicle platooning and the surrounding wireless-communications environment. 
monitoring of distances between the vehicles and of various operating parameters of the trucks forming the platoon.

In order to meet such strict requirements concerning reliability, exchange of information between the platooned trucks and the communication infrastructure (roadside units, small cells or macro cells) is required to efficiently manage prospective interference, both between individual platoons, and between the platoons and external sources of interference.

This paper focuses primarily on the impact that sensor inaccuracies (caused by imperfections) exert on the efficiency of V2X-aided platooning. In Section 2, we outline practical implementation of adaptive cruise control mechanisms. Section 3 presents an analysis of the impact that the quality of various sensors exerts on reliability and performance of the entire system. In Section 4, we discuss the main challenges related to platooning that relies on wireless communications, and provide a brief overview of the potential solutions improving reliability of the solution. Finally, Section 5 concludes the work.

\section{Implementation Issues}

Real-world implementation of the platooning technology is a big challenge. Driving needs to be controlled and coordinated with the use of sensors and actuators relying on a sophisticated algorithm. In addition, messages need to be exchanged between vehicles in the platoon, using wireless communications. The most important factors impacting the efficiency of platooning include the following: control system latency and accuracy of information collected by sensors. Cooperative adaptive cruise control (CACC) uses information both from local sensors and from other vehicles in the platoon (passed via a wireless link). CACC allows to boost platooning performance if, in addition to the requirement of a higher degree of accuracy of the control loop and of the sensors, wireless communication supports rapid, reliable and low-latency message exchange between the vehicles [4], [5].

The concept of autonomous driving, applied to a limited set of adjacent vehicles forming a platoon, has attracted a lot of interest in recent years. The CACC algorithm is proposed as a control mechanism for such a structure. Many researchers have been recently evaluating the performance of platooning that relied on the IEEE 802.11p standard for V2V communications. The SARTRE project [2] reported an experiment with two trucks and three cars forming a platoon. It has been proved that the platoon was able to drive at the maximum speed of $90 \mathrm{~km} / \mathrm{h}$, with the inter-vehicle distance ranging from 5 to $7 \mathrm{~m}$. On the other hand, the tests described in [3], [6] allowed to achieve the speed of $80 \mathrm{~km} / \mathrm{h}$ with the target inter-car distance equaling $10 \mathrm{~m}$. This was the result achieved during the Energy ITS project performed in Japan for a platoon of three fully-automated trucks moving on an expressway.

From the point of view of wireless communications, CACC suffers from insufficient reliability of message exchange be- tween the vehicles. A past study on the use of CACC while exchanging messages using the IEEE 802.11p standard revealed that even a non-significant increase in the density of vehicles on a highway may cause a congestion of the wireless channel. This will deteriorate performance of the CACC algorithm [7], [8]. One of the solutions of this problem is to use a dual-band transceiver that may operate simultaneously at two different frequencies (wireless channels). However, it should be noted that even if two ITS frequency channels are utilized, channel congestion is still possible if the scenario involves a high number of neighboring vehicles and a large amount of data to be exchanged. To solve this problem, the limit of two channels needs to be removed. This can be achieved by using the dynamic spectrum access technology, i.e. by relying on the cognitive radio concept. It is forecast that such an approach will be capable of improving the reliability of wireless communications and, indirectly, the performance of CACC used for vehicle platooning.

\section{Role of Sensors in Wireless Communications within Platoons}

On-board sensors constitute an essential part of a CACCcontrolled vehicle system. They measure the basic physical quantities necessary for the proper operation of the control algorithm. Sensors used in a vehicle may be divided into two types. The first category is used to monitor the vehicle's environment, while sensors of the other type are responsible for the evaluation of the vehicle itself. The first class includes long/medium range radar (LRR/MRR) sensors and forward collision warning (FCW) sensors. They determine the distance to the preceding vehicle and its instantaneous speed. The other group includes sensors that determine the instantaneous speed and acceleration of the vehicle in which they are installed.

\subsection{Sensor Inaccuracies}

Sensor inaccuracies may be determined in different ways. The significance of sensor errors may be described using the following categories: absolute accuracy, error distribution, error values corresponding to a given percentile of error distribution or output noise.

In order to add inaccuracies of the analyzed sensors to the system under consideration, it is assumed that sensor inaccuracies may be modeled using normal distribution truncated to $\pm 3 \sigma$. For the sensor accuracy distribution model in question, standard deviation that corresponds to a given sensor error has been determined. For the purpose of this paper, we investigated some off-the-shelf, average quality units that are installed or could be installed as onboard sensors.

The sensors investigated may be divided into the following groups, based on the parameter they measure: distance, relative velocity, ego velocity and acceleration. Depending on 
their tasks, these sensors may be characterized by different ranges and accuracy levels. The task of distance sensors is to determine distances to adjacent objects, i.e. moving vehicles and stationary obstacles. Relative speed sensors evaluate the speed of the surrounding vehicles with respect to the ego vehicle. In addition to sensors that monitor the position of adjacent vehicles, sensors that determine the speed and acceleration of the vehicle on which they are mounted are also required. For measuring the velocity of the ego vehicle, electro-mechanical sensors may be used, with their accuracy depending on the quality of the vehicle's tires.

\subsection{Sensors Measurements}

It is the objective of the distance sensors to measure range to adjacent moving vehicles or obstacles. Sensors offer different ranges and measurement accuracies, depending on their purpose. Table 1 presents a set of commercially available units with information taken from catalog notes, measurement reports, and scientific papers, presenting their features. The tables present designated model parameters as well.

Table 1

Sensors model

\begin{tabular}{|c|c|c|}
\hline Sensor reference & Accuracy & Std. dev. \\
\hline \multicolumn{3}{|c|}{ Distance sensors } \\
\hline [9] & $92 \%=0.5 \mathrm{~m}$ & 0.2860 \\
\hline [10] - Type 1 & $90 \%=0.6 \mathrm{~m}$ & 0.3650 \\
\hline [10] - Type 2 & $90 \%=1.5 \mathrm{~m}$ & 0.9120 \\
\hline [10] - Type 3 & $90 \%=0.9 \mathrm{~m}$ & 0.5470 \\
\hline SRR contin. [11] & Accuracy $=0.2 \mathrm{~m}$ & 0.0667 \\
\hline \multicolumn{3}{|c|}{$\begin{array}{c}\text { Relative speed sensors } \\
\end{array}$} \\
\hline LLR3 Bosch [12] & Accuracy $=0.12 \mathrm{~m} / \mathrm{s}$ & 0.0500 \\
\hline MRR Bosch [13] & Accuracy $=0.11 \mathrm{~m} / \mathrm{s}$ & 0.0367 \\
\hline$[9]$ & $85 \%=1.11 \mathrm{~m} / \mathrm{s}$ & 0.7700 \\
\hline [10] - Type 1 & $90 \%=0.25 \mathrm{~m} / \mathrm{s}$ & 0.1520 \\
\hline [10] - Type 2 & $90 \%=0.23 \mathrm{~m} / \mathrm{s}$ & 0.1400 \\
\hline$[10]-$ Type 3 & $90 \%=0.23 \mathrm{~m} / \mathrm{s}$ & 0.1400 \\
\hline \multicolumn{3}{|c|}{ Acceleration sensors } \\
\hline VBOX [14] & Accuracy $=0.3924 \mathrm{~m} / \mathrm{s}^{2}$ & 0.1308 \\
\hline SMB type 225 [15] & Output noise $=3.5 \mathrm{mg}$ & 0.0343 \\
\hline SMB type 227 [16] & Output noise $=5.0 \mathrm{mg}$ & 0.0491 \\
\hline Kistler [17] & $90 \%=0.23 \mathrm{~m} / \mathrm{s}^{2}$ & 0.0123 \\
\hline
\end{tabular}

Relative speed sensors that evaluate the velocity of surrounding vehicles constitute another group of measuring devices. As in the case of distance sensors, specific parameters and accuracies of those sensors are shown in Table 1.

Additionally to sensors that monitor the position of adjacent vehicles, CACC requires information on the speed and acceleration of the ego vehicle. In the case of ego vehicle velocity measurement, two options may be considered: electromechanical (accuracy depends on the quality of the vehicle's tires) or GPS-based devices.

To ensure a state-of-the-art approach to velocity measurement, electro-mechanical sensors are taken into account. For the purpose of simulations, it has been assumed that the device will be an electro-mechanical sensor with accuracy that is proportional to the actual speed of the vehicle, with the proportionality factor of $k=1.0 \%$ (the measured value is higher than the actual one).

The CACC formula considered takes also into account the acceleration rate of the platoon leader and the preceding car, so acceleration sensors should be used as well. For acceleration measurements, only high-precision GPS-based sensors are used (Table 1).

Due to the large number of devices to be tested, sensors representing the specific parameters (mean value and standard deviation) were identified. Mean errors and standard deviation values for all tested sensors are summarized in Table 2.

Table 2

Parameters of the sensors used

\begin{tabular}{|l|c|c|}
\hline \multicolumn{1}{|c|}{ Sensor } & Mean error & Standard deviation \\
\hline Distance sensor & 0 & 0.2794 \\
Rel. velocity sensor & 0 & 0.1439 \\
Velocity sensor & $1 \%$ & 0 \\
Acceleration sensor & 0 & 0.0410 \\
\hline
\end{tabular}

\subsection{Simulation Model}

Before we present the results of our simulation investigations aimed at determining the influence of imperfect sensors on the platoon's performance, we discuss the simulation assumptions, platoon structure, as well as the parameters of the so-called jamming vehicle considered in the simulations. The role of the jammer vehicle is to disturb the fluent movement of the platoon. Its velocity varies in accordance with a predefined pattern in 30-second cycles (looped for the course of the simulation). Each cycle lasts $30 \mathrm{~s}$ and consists of three phases: braking (speed decreases from $130 \mathrm{~km} / \mathrm{h}$ to $30 \mathrm{~km} / \mathrm{h}$ ), acceleration (acceleration factor equal to $1.5 \mathrm{~m} / \mathrm{s}^{2}$, with the speed reaching $130 \mathrm{~km} / \mathrm{h}$ again) and, finally, constant speed travel for approximately $2.04 \mathrm{~s}$.

\subsection{Actuation Lag}

After the acceleration value has been calculated by the ACC/CACC controller, it needs to be used by the system, This, however, is not done instantaneously, but with a delay referred to as actuation lag. In the simulator, this delay is modeled as a first order low-pass filter applied to the output of the cruise controller:

$$
P(s)=\frac{1}{(\tau s+1)},
$$


where $\tau$ is the time constant of the filter. In [18], a list of factors that affect actuation lag in the case of a humandriven vehicle is presented. Taking all these factors into account, we can conclude that time constant $\tau$ could be as high as $500 \mathrm{~ms}$.

Recent papers [19], [20] indicate that in the case of humandriven vehicles the total lag value could be decreased to approx. $200 \mathrm{~ms}$. Moreover, a further decrease in actuation lag to a few tens of milliseconds is expected [8]. Therefore, two different actuation lag values, i.e. 20 and $200 \mathrm{~ms}$, are investigated here.

\subsection{Simulation Assumptions}

In the considered scenario, we investigated a homogeneous platoon traveling in one lane of a highway. The first car in the platoon is the so-called leader and is preceded by another vehicle that interrupts the fluent movement of the platoon - the so-called jammer. The speed of the jammer changes (periodic breaking and acceleration), affecting the platoon's movement. With the help of control mechanisms, vehicles in the platoon attempt to respond to the jammer's behavior by adjusting their speeds and accelerations, in order to maximize platoon performance (i.e. they attempt to minimize the length of the platoon) and to avoid crashes. Within the platoon, two controlling algorithms are considered. One is the adaptive cruise control (ACC) algorithm (used by the platoon leader) and the other once is the CACC algorithm used by the remaining vehicles.

In the ACC algorithm, the speed of and distance to the preceding car are measured by built-in (or onboard) sensors, while in CACC, in addition to sensor measurements, messages from other vehicles that are transmitted by radio are relied upon. In this work, communication between the vehicles is performed based on the IEEE 802.11p standard.

\subsection{Simulation Parameters}

The set of parameters valid for all simulation runs and the configurations parameters used in IEEE 802.11p are presented in Table 3.

In the simulation, sensor inaccuracies are investigated separately to determine their impact on the platoon's performance. Then, all inaccuracies considered are combined to obtain the overall impact of all inaccuracies on the platoon's performance.

The performance of the platoon with the sensors adopted was evaluated in terms of mean inter-vehicle spacing. Intervehicle spacing corresponds to the bumper-to-bumper distance measured, averaged over time, for all pairs of subsequent vehicles in the platoon, and for all simulation runs over the chosen target CACC distance. It is expected that the average inter-vehicle spacing closely approximates the target CACC distance, i.e. the minimum target distance that guarantees collision-free performance of the platoon.

Moreover, it needs to be mentioned that we used, in the simulation, an improved version of the CACC algorithm introduced in [8], referred to as modified CACC, offering
Table 3

Simulation and IEEE 802.11 p configuration parameters

\begin{tabular}{|l|c|}
\hline \multicolumn{1}{|c|}{ Parameter } & Value \\
\hline \hline Number of simulation runs & 100 \\
Simulation time & $900 \mathrm{~s}$ \\
Platoon size & $10 \mathrm{cars}$ \\
Engine actuation lag & $20 / 200 \mathrm{~ms}$ \\
ACC headway time & $0.2 \mathrm{~s}$ \\
BSM message size & 300 bytes \\
CACC message size & 16 bytes \\
Spectrum band & ITS-G5A(5895-5905 MHz) \\
Channel estimation & ITU Vehicular-A \\
Channel model & Winner+ B1 LOS \\
Path loss model & $3.5 \mathrm{~m}$ \\
Antenna height & Log-normal \\
Shadowing distribution & $3 \mathrm{~dB}$ \\
Shadowing std. dev. & $25 \mathrm{~m}$ \\
Decorrelation distance & $23 \mathrm{dBm} / \mathrm{MHz}$ \\
TX power density & $-174 \mathrm{dBm} / \mathrm{Hz}$ \\
Noise power & $9 \mathrm{~dB}$ \\
Noise figure & $1 / 1$ \\
TX/RX antennas & Off \\
Power control & QPSK, $R=1 / 2$ \\
MCS &
\end{tabular}

significantly better performance that is reflected by lower mean inter-vehicle distances.

\subsection{Sensors Inaccuracies}

The first part of the investigations performed is related to the impact of sensor quality on the behavior of the platoon. Figures 2 and 3 show the mean distances between platoon vehicles, assuming that the vehicles are equipped with distance and velocity sensors used in the CACC algorithm. These distances are calculated as the average of all bumper-to-bumper distances between the vehicles in the platoon, measured throughout the entire simulation period. The results are compared with the reference values which correspond to the situation in which all sensors work perfectly.

The introduction of inaccuracies results in a slight degradation of the platoon's performance. The said degradation equals approx. $0.2 \mathrm{~m}$ for the distance sensor and $0.4 \mathrm{~m}$ for the speed sensor in the case of the $20 \mathrm{~ms}$ scenario, and $0.3 \mathrm{~m}$ for the distance sensor and $0.15 \mathrm{~m}$ for the speed sensor in the case of the $200 \mathrm{~ms}$ scenario.

Another sensor that has been analyzed is the in-vehicle speed sensor. The mean distances obtained for different actuation lag values are shown in Fig. 4.

Based on the above results, it can be clearly stated that in the case of velocity sensor, the inaccuracies exert a nega- 


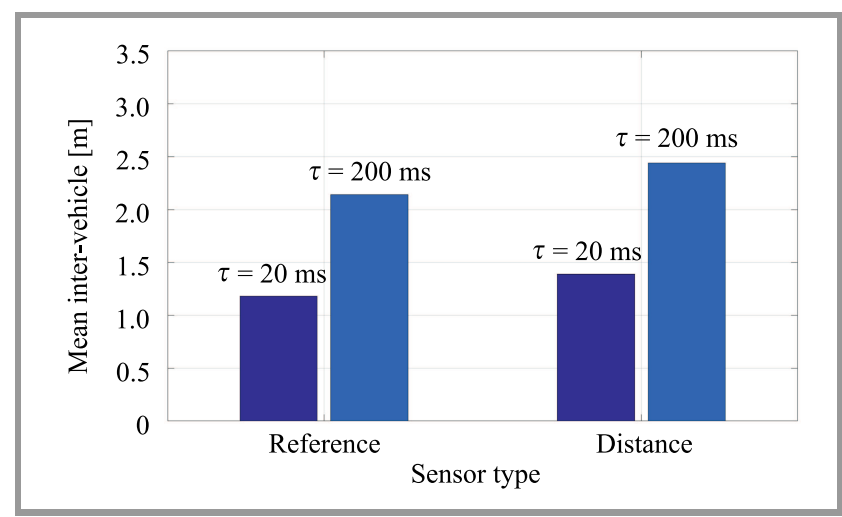

Fig. 2. Mean platoon inter-vehicle distance for distance sensors used in the CACC algorithm.

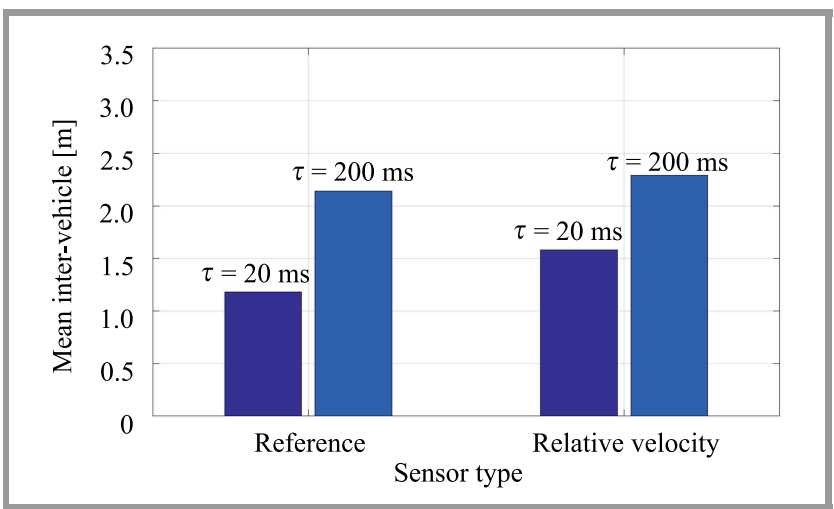

Fig. 3. Mean platoon inter-vehicle distance for relative speed sensors used in the CACC algorithm.

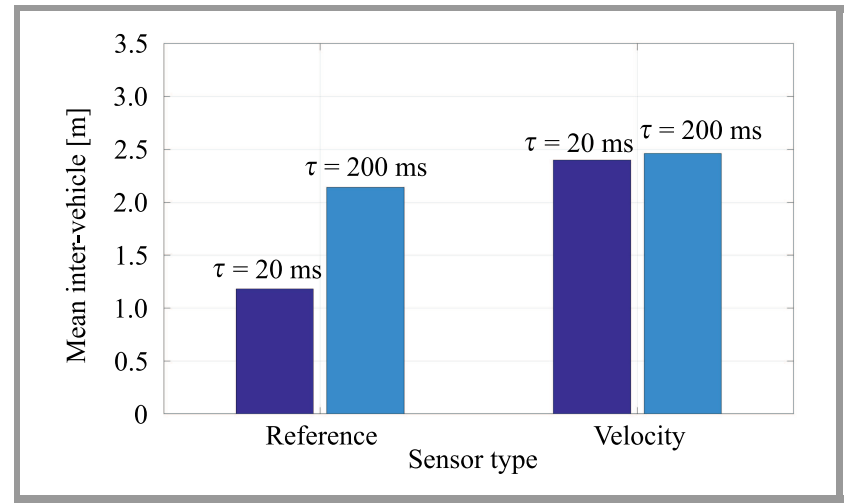

Fig. 4. Mean platoon inter-vehicle distance for speed sensors used in the CACC algorithm.

tive impact on the behavior of the platoon. This impact is reflected by a significantly higher mean distance between vehicles, which increases by $1.28 / 0.26 \mathrm{~m}$ for $20 / 200 \mathrm{~ms}$ scenarios, respectively.

The last device (Fig. 5) that has been analyzed is the vehicle acceleration sensor which determines the instantaneous acceleration factor of the ego vehicle. According to the literature, as such a unit should operate based on GPS data, it may achieve very good parameters. Tests performed with such an acceleration sensor show that the target distance

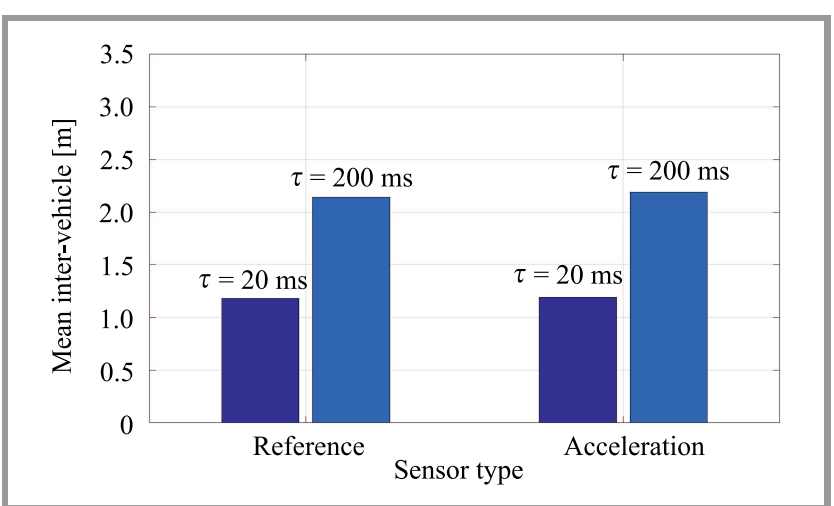

Fig. 5. Mean platoon inter-vehicle distance for acceleration sensors used in the CACC algorithm.

is only slightly affected, even for the lowest-quality sensor. Moreover, the mean inter-vehicle distance deterioration observed is as low as $0.01 \mathrm{~m}$, compared with the reference values.

Although the presented acceleration sensors are characterized by great accuracy, their largest drawback is that they need to be within range of the GPS signal. Thus, these sensors might experience some difficulties in the so-called urban canyon scenarios or in tunnels. To overcome this, additional post-processing of the velocity data will be required.

Finally, the combined effect of all sensors on the behavior of the platoon was also analyzed, and distances between vehicles within the platoon were determined (Fig. 6). The results obtain clearly indicate that the quality of the devices used exerts a great impact on the platoon. Even the best quality units affect the platoon performance significantly, causing an increase in the observed mean distance between vehicles, equaling one meter.

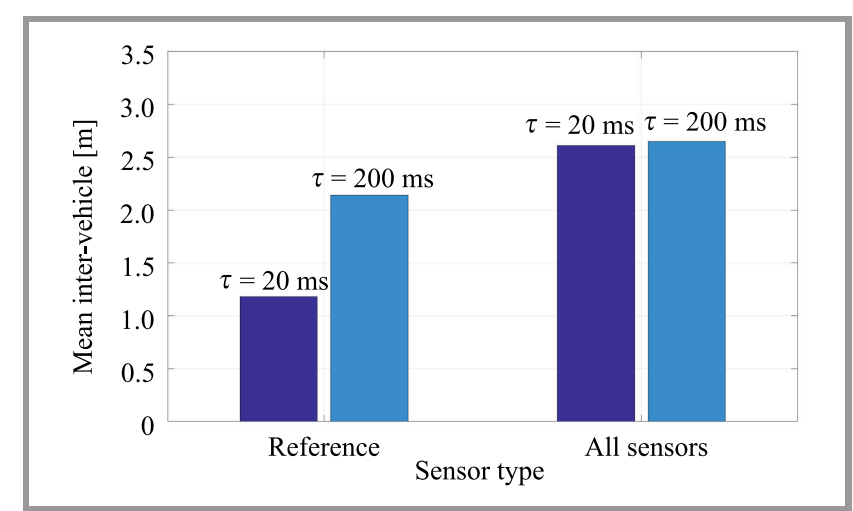

Fig. 6. Mean platoon inter-vehicle distance for all sensors used in the CACC algorithm.

\section{Challenges Affecting Wireless Communications}

V2V communications are usually proposed to be performed using cellular networks (e.g. LTE, 5G) or IEEE 802.11p 
systems. However, it was observed that these technologies use fixed wireless channels, which lowers reliability when a high number of users (cars, trucks, platoons) is present.

A potential solution is to base intra-platoon communications on the so-called white spaces, i.e., frequencies that are unused by the licensed system at a given location, at the time of the platoon's arrival, such as the cognitive radio concept. This approach to spectrum usage is known as dynamic spectrum access (DSA). Its application is motivated by the number of spectrum occupancy measurements carried around the world. It has been revealed that $50 \%$ to $80 \%$ frequencies in the range of up to $6 \mathrm{GHz}$ are unused. Therefore, potential frequency resources that may be reused exist. If a given frequency band is currently not occupied by a specific licensed system, it may be used for secondary system transmissions (without a license to use this band) [21]. In our case, the role of the secondary system may be played be the platoon of vehicles. Moreover, the higher the frequency, the more white spaces may be expected. Carrier frequencies of above $6 \mathrm{GHz}$ and DSA may be considered for short-range V2V communications [22].

In the case of $\mathrm{V} 2 \mathrm{~V}$ communications, access to timely and accurate information about the surrounding environment may be very important. When vehicles are aware of the status of other objects on the road ahead of them, they can change the route or at least modify the driving parameters to reduce fuel consumption. Decreasing the probability of a crash is of the highest importance. However, in the context of DSA for V2V communications, availability of specific context information databases is required. The databases will store various data structures depending on the applications. In order to enable efficient management of the spectrum resources, information about transmissions using licensed systems and abut secondary users (platoons utilizing DSA) is needed. A scenario may be imagined in which the platoon leader communicates with the database providing suggestions as to which frequency band should be used, taking into account interference received from and caused to other platoons. However, information about spectrum usage is the simplest of potential applications of such a database. It is envisaged that context information databases may store much more information, with their range varying from the number of trucks in each convoy, to their speed, to the current length of the platoon. This may be a source of typical speeds achieved on a given road depending on weather conditions. The structure of the database system should be, most likely, hierarchical. Longterm information about the environment may be stored in remote databases, while short-term information is stored in their local counterparts [23].

\subsection{Sensor Inaccuracy vs. Information Available in Databases}

As shown in Section 3, sensor inaccuracy directly impacts the performance of CACC. Error-affected information pro- vided by the sensors, combined with limited reliability of wireless links may render effective platooning impossible due to an excessive number of errors/variances in the information fed to the CACC control algorithm. Therefore, novel solutions have to be proposed, such as dynamic spectrum access for $\mathrm{V} 2 \mathrm{~V}$ transmissions. If raw sensing information is to be exchanged between vehicles, the $\mathrm{mm}$ wave band, offering a higher bandwidth, may be considered [22], [24]. In such a case, throughput required along the links between vehicles may increase to hundreds of Mbps, while requirements concerning latency and reliability remain the same. Although the millimeter wave band allows for wider bandwidth, it poses serious challenges as well, e.g. reduces the transmission range. Therefore, lower frequency bands may be considered for intra-platoon communications as well [22]. In any frequency band of choice, a dynamic spectrum management mechanism is needed enable the selection of a frequency band that guarantees the best possible transmission conditions. The spectrum management mechanism may consider a licensed spectrum for inter-platoon communications as well. Proper selection of the frequency band may be aided with context information stored in dedicated databases. Multiple parameters stored therein may be used to improve the frequency selection procedure.

\section{Conclusions}

This article discusses various aspects of vehicle platooning, in particular the impact that inaccuracy of onboard sensors on the performance of a CACC-based platoon. Additionally, it considers sensing inaccuracy and limited reliability of wireless communications. Given the limited accuracy of the sensors, challenges for future V2V wireless communication have been formulated, including restrictions concerning delay, reliability and perceived collaboration. A suggested potential solution to increase CACC reliability is to use dynamic allocation of numerous frequency bands, including the millimeter wave band, to improve data transmission speed and to reduce the load of current ITS bands.

\section{Acknowledgements}

The work has been performed under project no. 2018/29/ B/ST7/01241 funded by the National Science Center in Poland.

\section{References}

[1] A. Osseiran et al., "Scenarios for 5G mobile and wireless communications: the vision of the METIS project", IEEE Commun. Mag., vol. 52, no. 5, pp. 26-35, 2014 (DOI: 10.1109/MCOM.2014.6815890).

[2] E. Chan, "Overview of the SARTRE platooning project: Technology leadership brief”, SAE Technical Paper 2012-01-9019, 2012 (DOI: 10.4271/2012-01-9019). 
[3] S. Tsugawa, S. Kato, and K. Aoki, "An automated truck platoon for energy saving", in Proc. IEEE/RSJ Int. Conf. on Intell. Robots and Sys., San Francisco, CA, USA, 2011 pp. 4109-4114 (DOI: 10.1109/IROS.2011.6094549).

[4] J. Ploeg et al., "Design and experimental evaluation of cooperative adaptive cruise control", in Proc. of the 14th Int. IEEE Conf. on Intell. Transport. Syst. ITSC 2011, Washington, DC, USA, 2011, pp. 260-265 (DOI: 10.1109/ITSC.2011.6082981).

[5] D. Jia et al., "A survey on platoon-based vehicular cyber-physical systems", IEEE Commun. Surv. Tutor., vol. 18, no. 1, pp. 263-284, 2016 (DOI: 10.1109/COMST.2015.2410831).

[6] S. Tsugawa, S. Jeschke, and S. E. Shladover, "A review of truck platooning projects for energy savings", IEEE Trans. on Intell. Veh., vol. 1, no. 1, pp. 68-77, 2016 (DOI: 10.1109/TIV.2016.2577499).

[7] P. Sroka et al., "Szeregowanie transmisji wiadomości typu BSM w celu poprawy działania kooperacyjnego adaptacyjnego tempomatu (Improvement of cooperative adaptive cruise control operation by scheduling of BSM messages)", Przeglad Telekomunikacyjny+ Wiadomości Telekomunikacyjne, vol. 2017, no. 6, pp. 350-355, 2017 (DOI 10.15199/59.2017.6.45) [in Polish].

[8] M. Sybis et al., "Communication aspects of a modified cooperative adaptive cruise control algorithm", IEEE Trans. on Intell. Transport. Syst., vol. 20, no. 12, pp. 4513-4523, 2019 (DOI: 10.1109/TITS.2018.2886883).

[9] "Overview of Data Accuracy Evaluations for STMS Vehicle Detectors", Tech. Rep., Sensebit AB, Dec. 2012 [Online]. Available: http://sensebit.se/wp-content/uploads/2015/02/Overview-of-dataaccuracy-evaluations-for-STMS-vehicle-detectors-2013.pdf

[10] F. de Ponte Müller, E. Diaz, and I. Rashdan, "Cooperative positioning and radar sensor fusion for relative localization of vehicles", in Proc. of the 2016 IEEE Intell. Veh. Symp. IV 2016, Gothenburg, Sweden, 2016 (DOI: 10.1109/IVS.2016.7535520).

[11] "Short range radar - SRR320", Continental AG [Online]. Available: https://www.continental-automotive.com/en-g1/2-Wheeler/ Safe-Mobility/Sensors/Short-Range-Radar-SRR320

[12] "Long-range radar sensor", Bosch Mobility Solutions [Online]. Available: https://www.bosch-mobility-solutions.com/en/productsand-services/passenger-cars-and-light-commercial-vehicles/driverassistance-systems/left-turn-assist/long-range-radar-sensor/

[13] "Mid-range radar sensor (MRR rear)", Bosch Mobility Solutions [Online]. Available: https://www.bosch-mobility-solutions.com/ en/products-and-services/passenger-cars-and-light-commercialvehicles/driver-assistance-systems/lane-change-assist/mid-rangeradar-sensor-mrrrear/

[14] "Speed Sensor Range (VBSSxx/VBSS100SL)", Racelogic Limited [Online]. Available: https://racelogic.support/

01VBOX_Automotive/02Speed_Sensors/Single_and_Dual_ Antenna_Speed_Sensors

[15] "BMA225, Digital, triaxial acceleration sensor", Bosch Sensortec [Online]. Available: https://ae-bst.resource.bosch.com/ media/_tech/media/product_flyer/BST-BMA255-FL000.pdf

[16] "SMB227, Low-g Accelerometer for Vehicle Dynamics Control", Bosch Sensortec [Online]. Available: https://www.arrow.com/it-it/ datasheets/8958509520/bosch/smb227

[17] "Capacitive MEMS, Single-Axis Accelerometer Type 8315A...", KISTLER Acceleration Sensor", Kistler Group, Switzerland [Online]. Available: http://www.helmar.com.pl/helmar/plik/ pliki-produktow-kistler_8315_nn4910.pdf

[18] R. Rajamani, Vehicle Dynamics and Control. Springer, 2012 (ISBN: 9781461414322).

[19] M. Wang et al., "Delay-compensating strategy to enhance string stability of adaptive cruise controlled vehicles", Transportmetr. B: Transp. Dynam., vol. 6, no. 3, pp. 211-229, 2018 (DOI: 10.1080/21680566.2016.1266973).

[20] F. A. Mullakkal-Babu, M. Wang, B. van Arem, and R. Happee, "Design and analysis of full range adaptive cruise control with integrated collision avoidance strategy", in Proc. IEEE 19th Int. Conf. on Intell. Transport. Syst. ITSC 2016, Rio de Janeiro, Brazil, 2016, pp. 308-315 (DOI: 10.1109/ITSC.2016.7795572).
[21] T. Yucek and H. Arslan, "A survey of spectrum sensing algorithms for cognitive radio applications", IEEE Commun. Surv. Tutor. vol. 11, no. 1, pp. 116-130, 2009 (DOI: 10.1109/SURV.2009.090109).

[22] J. Choi et al., "Millimeter-wave vehicular communication to support massive automotive sensing", IEEE Commun. Mag., vol. 54, no. 12, pp. 160-167, 2016 (DOI: 10.1109/MCOM.2016.1600071CM).

[23] J. Perez-Romero et al., "On the use of radio environment maps for interference management in heterogeneous networks", IEEE Commun. Mag., vol. 53, no. 8, pp. 184-191, 2015 (DOI: 10.1109/MCOM.2015.7180526).

[24] S. Lien et al., "Latency-optimal mmWave radio access for V2X supporting next generation driving use cases", IEEE Access, vol. 7, pp. 6782-6795, 2019 (DOI: 10.1109/ACCESS.2018.2888868).

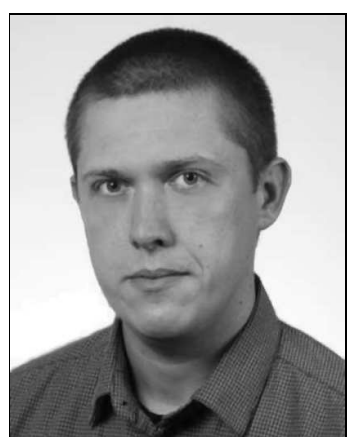

Michat Sybis received his M.Sc. in Electronics and Telecommunications and Ph.D. (with honors) in Telecommunications from Poznań University of Technology, Poznan, Poland, in 2007 and 2012, respectively. $\mathrm{He}$ is currently an Assistant Professor at the Institute of Radiocommunications, Faculty of Electronics and Telecommunications at Poznan University of Technology. His research interests include coding techniques, iterative decoding, 5G mobile wireless systems and vehicular communications.

(D) https://orcid.org/0000-0002-7149-2386

E-mail: michal.sybis@put.poznan.pl

Institute of Radiocommunications

Faculty of Electronics and Telecommunications

Poznań University of Technology

Polanka 3

60-965 Poznan, Poland

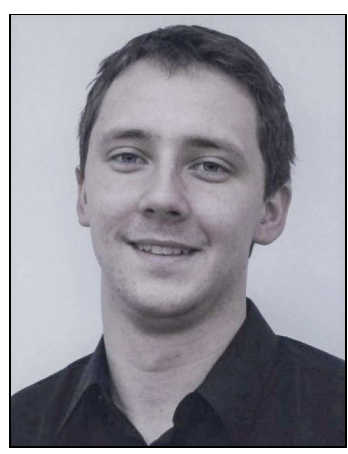

Paweł Sroka received his M.Sc. in 2004 and Ph.D. in Telecommunications (with honors) in 2012 from Poznan University of Technology (PUT), Poland. $\mathrm{He}$ is employed at the Institute of Radiocommunications, PUT, as an Assistant Professor. $\mathrm{He}$ participated in numerous international and national research projects in the area of wireless communications. His main research interests include V2X communications and radio resource management for wireless networks and MIMO systems.

(iD) https://orcid.org/0000-0003-0553-7088

E-mail: pawel.sroka@put.poznan.pl

Institute of Radiocommunications

Faculty of Electronics and Telecommunications

Poznan University of Technology

Polanka 3

60-965 Poznan, Poland 


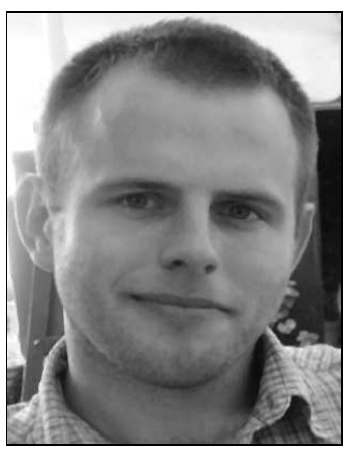

Paweł Kryszkiewicz received his M.Sc. and Ph.D. degrees (with honors) in Telecommunications from Poznan University of Technology (PUT), Poland, in 2010 and 2015, respectively, where he is currently an Assistant Professor at the Institute of Radiocommunications. He was involved in a number of national and international projects. His main research interests include problems concerning the physical layer of cognitive radio systems, multicarrier signal design for green communications, and interference limitation in $5 \mathrm{G}$ systems.

(iD) https://orcid.org/0000-0001-9054-9416

E-mail: pawel.kryszkiewicz@put.poznan.pl Institute of Radiocommunications

Faculty of Electronics and Telecommunications

Poznan University of Technology

Polanka 3

60-965 Poznan, Poland 